\title{
AN ON-LINE MULTIWAY APPROACH TO IN-SITU NDI LOOKING AT THE PZL-130TCII
}

\author{
Krzysztof Dragan ${ }^{1}$ \\ Michał Dziendzikowski \\ Artur Kurnyta ${ }^{1}$ \\ Adam Latoszek ${ }^{1}$ \\ Andrzej Leski ${ }^{1}$ \\ Sylwester Kłysz ${ }^{1,2}$ \\ ${ }^{1}$ Air Force Institute of Technology, Warsaw, Poland \\ ${ }^{2}$ University of Warmia and Mazury, Olsztyn, Poland
}

\begin{abstract}
Providing a reliable and universal Structural Health Monitoring (SHM) system allowing for remote aircraft inspections and a reduction of maintenance costs is a major challenge confronting the aerospace industry today. SHM based on guided Lamb waves is one of the approaches capable of addressing the issue while satisfying all the associated requirements. This paper presents a holistic approach to the continuous real time damage growth monitoring and early damage detection in aircraft structure. The main component of the system is a piezoelectric transducers (PZT) network. It is complemented by other SHM methods: Comparative Vacuum Monitoring $\left(C V M^{T M}\right)$ and Resistance Gauges at selected aircraft hot spots. The paper offers the description of damage detection capabilities including the analysis of data collected from the PZL-130 Orlik aircraft full-scale fatigue test.
\end{abstract}

Keywords: Structural Health Monitoring, PZT Sensor Network, Fatigue Crack Detection, Full Scale Fatigue Test

\section{INTRODUCTION}

The problem of monitoring aircraft structural health throughout the operational phase (operation and maintenance) is very complex and challenging. The article presents an approach to building an integrated monitoring system validated for the aircraft operational phase. The aircraft used for the purposes of the research was the PZL ORLIK TC II, a turbo propeller training aircraft operated by the armed forces. This aircraft is primarily used in the preliminary phase of training of military pilots. Due to the structure modification the aircraft will undergo the full-scale fatigue test (FSFT). That test opens an opportunity for the installation of a SHM system at selected 'hot spot' locations to detect early damage. In the future, this technology may support or partly replace scheduled NDI inspections. The SHM system designed and installed on the aircraft is based on three different methods: Comparative Vacuum Monitoring - $\mathrm{CVM}^{\mathrm{TM}}$ (Fig. 1), Resistance Gauges RG (Fig. 2), Piezoelectric Transducers Network - PZT (Fig. 1, Fig. 2). In the most of the monitored aircraft hot spots, those systems were hybridized (Fig. 1, Fig. 2). They operated complementarily in order to enable comparing the levels of efficiency they achieved and improve damage detection capabilities alongside the false calls ratio. The structure was monitored remotely with the use of an on-line data acquisition unit and dedicated software (Fig. 3, Fig. 4). 


\section{SHM BASED ON GUIDED LAMB WAVES}

A brief overview of the SHM system for fatigue crack growth monitoring is presented in the following section. The system's building blocks are schematically presented in the picture (Fig. 5). These are:

- PZT network divided into several measuring nodes;

- Remote Monitoring Unit (RMU) - based on DSP architecture CPU;

- Data Storage Unit (DSU);

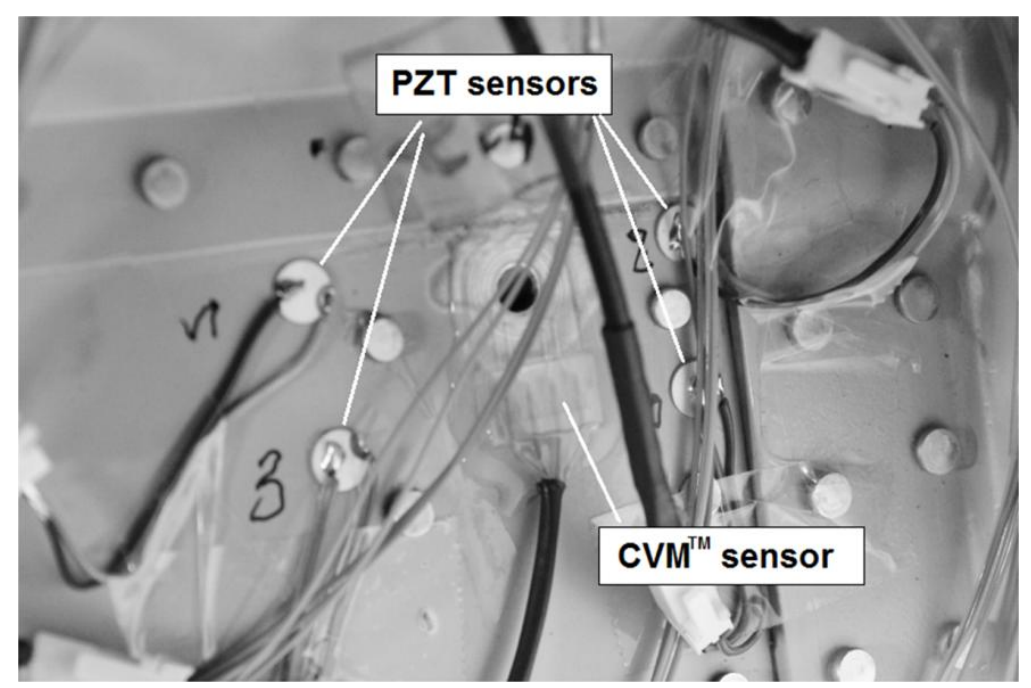

Fig. 1. CVM $M^{T M}$ and PZT sensors at an aircraft structure hot spot

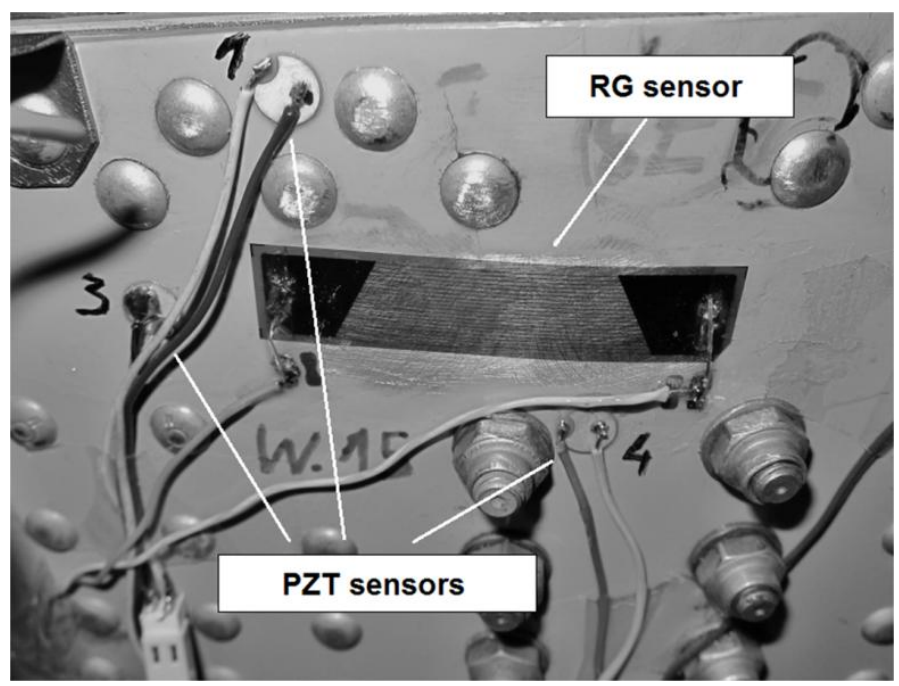

Fig. 2. RG and PZT sensors at an aircraft structure hot spot

- Graphical User Interface (GUI).

The core of the RMU consists of four subsequent routines:

- signal collecting and its storage in the DSU if indicated by a sensor self-diagnostic component; 
- signal processing based on several signal Damage Indices (DI) correlated with fatigue crack growth;

- sensor self-diagnostic component validating the PZT network, e.g. noise detection, sensors' surface coupling strength, significant sensor working conditions changes detection;

- data classification methods for damage growth assessment.

An issue of utmost importance in applying a PZT based monitoring system in structures used in aerospace is to ensure the durability of a sensor network in extremely varying environmental conditions. Thus a network self-diagnostic tool allowing for signal decoherence tracking in time is a vital component of any such application. Furthermore, as most data classification models are sensitive to outlying observations, efficient sensor self-diagnostics prior to crack growth assessment is crucial to the proper functioning of the system, e.g. the avoidance of misclassification.

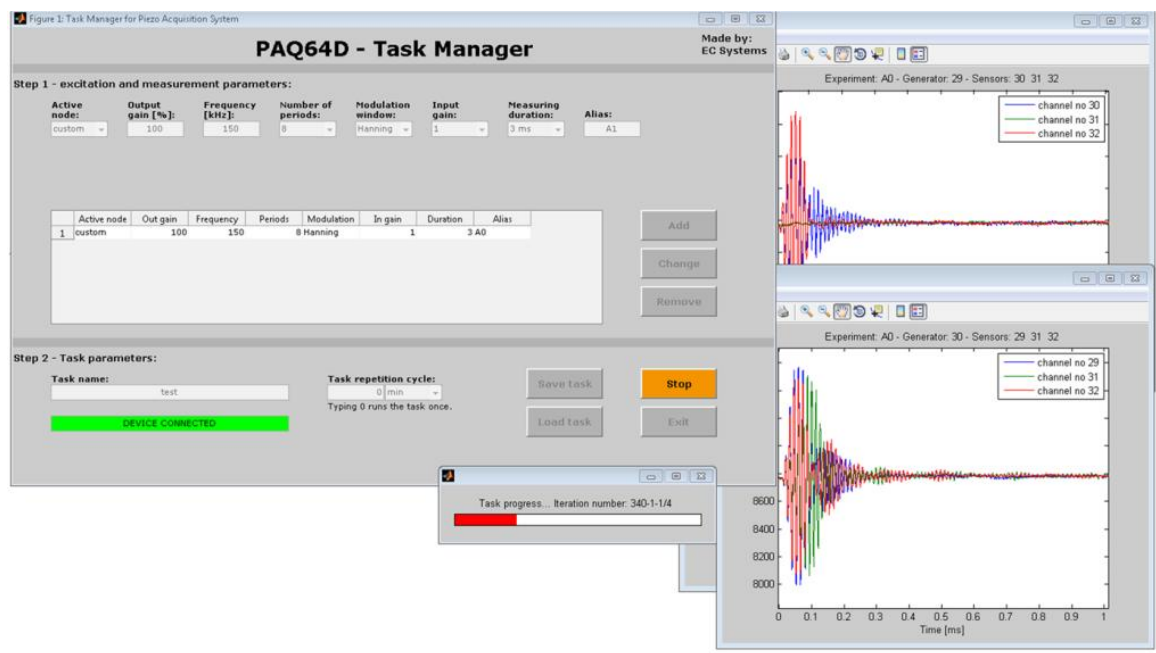

Fig. 3. Control panel of a PZT sensor network remote monitoring unit

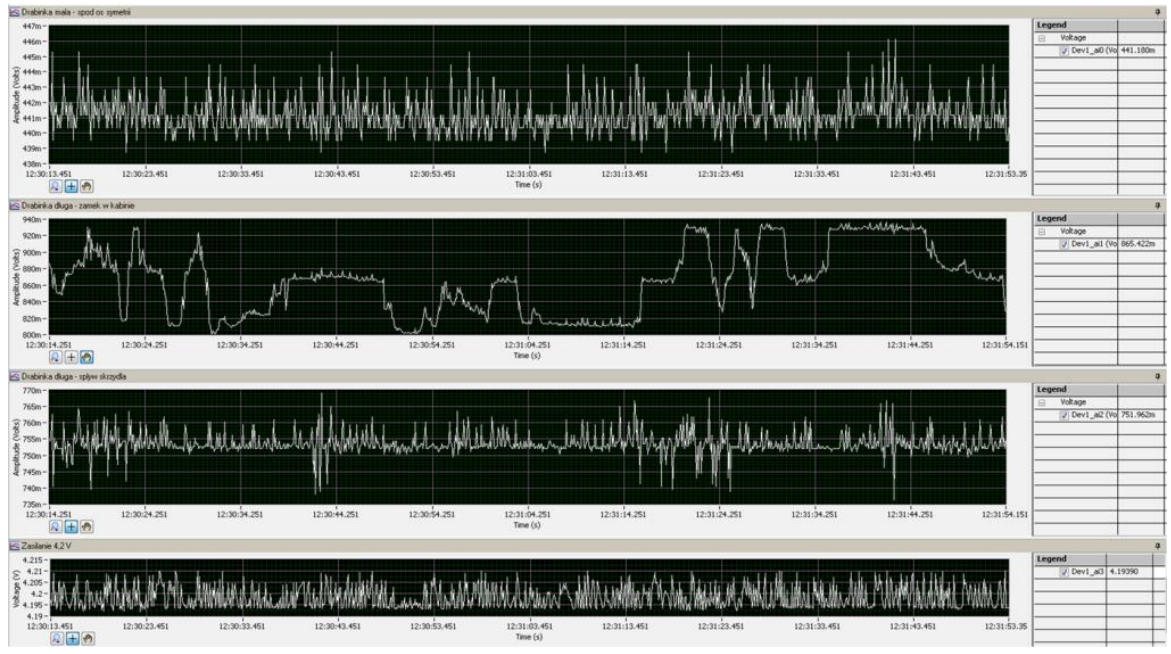

Fig. 4. RG sensors remote data acquisition unit 


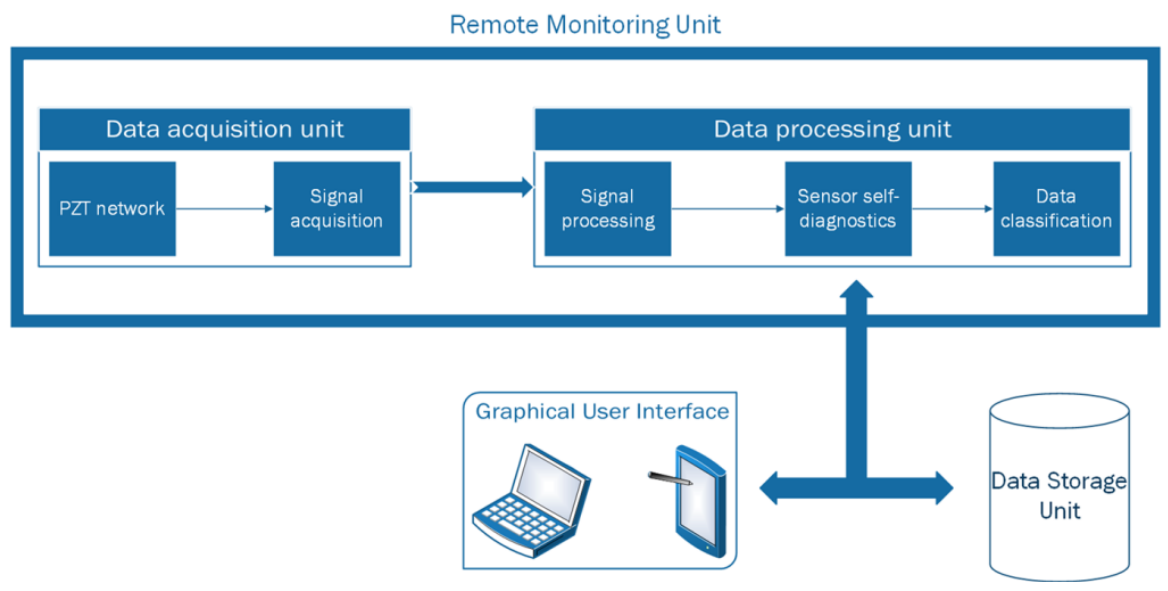

Fig. 5. SHM system block diagram

According to the proposed approach, signals which do not pass a sensor integrity check are stored in a DSU for further expert assessment as depicted in Figure 5.

The issue of damage monitoring with the use of elastic waves excitation in a given medium is currently being intensively studied. Depending on their source and the geometry of the structure under consideration, elastic waves can propagate over a significant distance. They are also sensitive to local structure discontinuities and deformations providing a tool for detecting local damage in large aerospace structures. A major obstacle to the direct application of this method, however, is the complexity of signals excited in real structures. Two examples of baseline signals obtained for structures of simple geometry and containing riveted joints and other wave reflectors are presented below (Fig. 6):
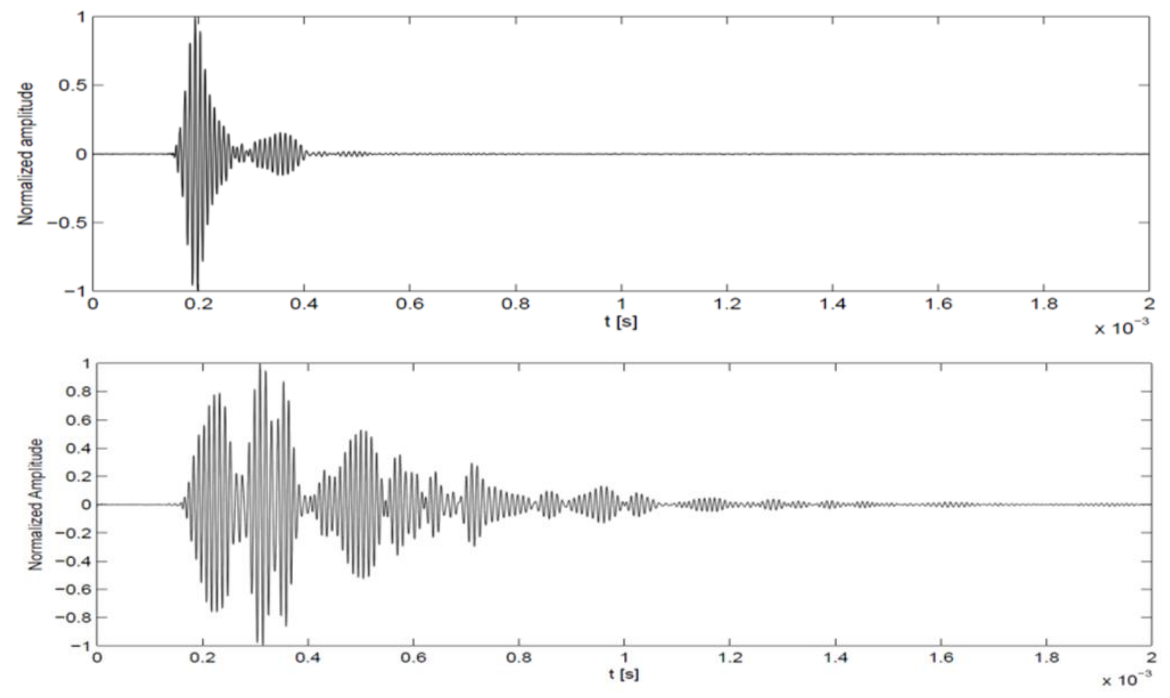

Fig. 6. Examples of reference signals, for structures of different types

For a given signal, information concerning the health of a structure is usually acquired by comparing it with a reference signal, i.e. the baseline, obtained for the initial state of the structure. In fully automated systems, this is usually done with the use of certain comparative signal characteristics called Damage Indices (DI). Denoting as $f_{g s}$ the signal generated in the transducer $g$ and received in the sensor $s$, and as $f_{g s, b}$ its baseline, i.e. the reference signal collected for the 
undamaged state of the structure, some basic DI can be defined using the following simple signal characteristics:

$$
\begin{aligned}
& L^{1} \text { symmetric characteristic } \quad-\quad D I_{1}(g, s)=\frac{\left|\int\left(\left|f_{g s}\right|-\left|f_{g s, b}\right|\right) d t\right|}{\int\left|f_{g s, b}\right| d t}, \\
& L^{2} \text { symmetric characteristic } \quad-\quad D I_{2}(g, s)=\frac{\left|\int\left(f_{g s}\right)^{2}-\left(f_{g s, b}\right)^{2} d t\right|}{\int\left(f_{g s, b}\right)^{2} d t} \text {, } \\
& \text { correlation with the baseline } \quad-\quad D I_{3}(g, s)=1-\operatorname{cor}(g, s) \text {. }
\end{aligned}
$$

Similar DI can be obtained using Fourier filtered signals, their envelopes or other signal transformations. These DI are correlated with the total energy received by a given sensor and can therefore capture the two main modes of the interaction between a guided wave and a fatigue crack. Low information content carried by those DI makes them more persistent under varying sensor working conditions. This should improve the false calls ratio of the system.

There are remarkable examples of applications of analogous DI to determine the localization of a damage [6-8]. In these methods, two stage algorithms are used. First, for each sensing path $g \rightarrow s$, i.e. a signal received in the sensor $s$ originated from the generator $g$, the structure is quantified into a damaged or undamaged state. This quantitative assessment can be performed using a threshold level of one or multiple DI. Then, if the structure is considered as damaged, a probability density of the damage localization in a given network cell is calculated. This density depends on DI values and the properly defined distance between a given point and the sensing path $g \rightarrow s$. Finally, joint probability for the damage localization is provided using probability maps obtained for all possible sensing paths in the network cell.

\section{MONITORING RESULTS}

In the following section, damage detection capabilities of the system are discussed based on two chosen hot spots monitored (Fig. 7). In both of locations Resistance Crack Gauges (RCG) adapted to the structure geometry were installed in order to verify the system indications. The crack was developing in the network shown in Figure 7(b) while the other hot-spot (Fig. 7(a)) remained undamaged. One of the network nodes (Fig. 7(a)) was installed on a structure containing riveted joints and other wave reflectors, the other structure (Fig. 7(b)) being of a relatively simple geometry. The Averaged Damage Indices for both of the nodes are presented in Figure 8. It was observed that the number of well-separated groups of data, corresponding to different crack sizes agrees with the number of sensing paths crossed by the crack. The data corresponding to subsequent periods of the monitoring of the undamaged structure are not separated (Fig. 8).

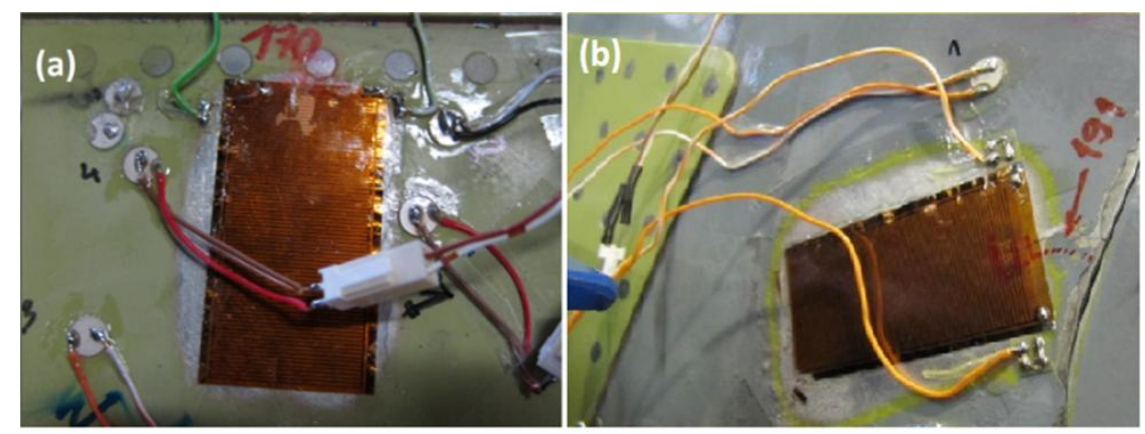

Fig. 7. Monitored hot-spots 

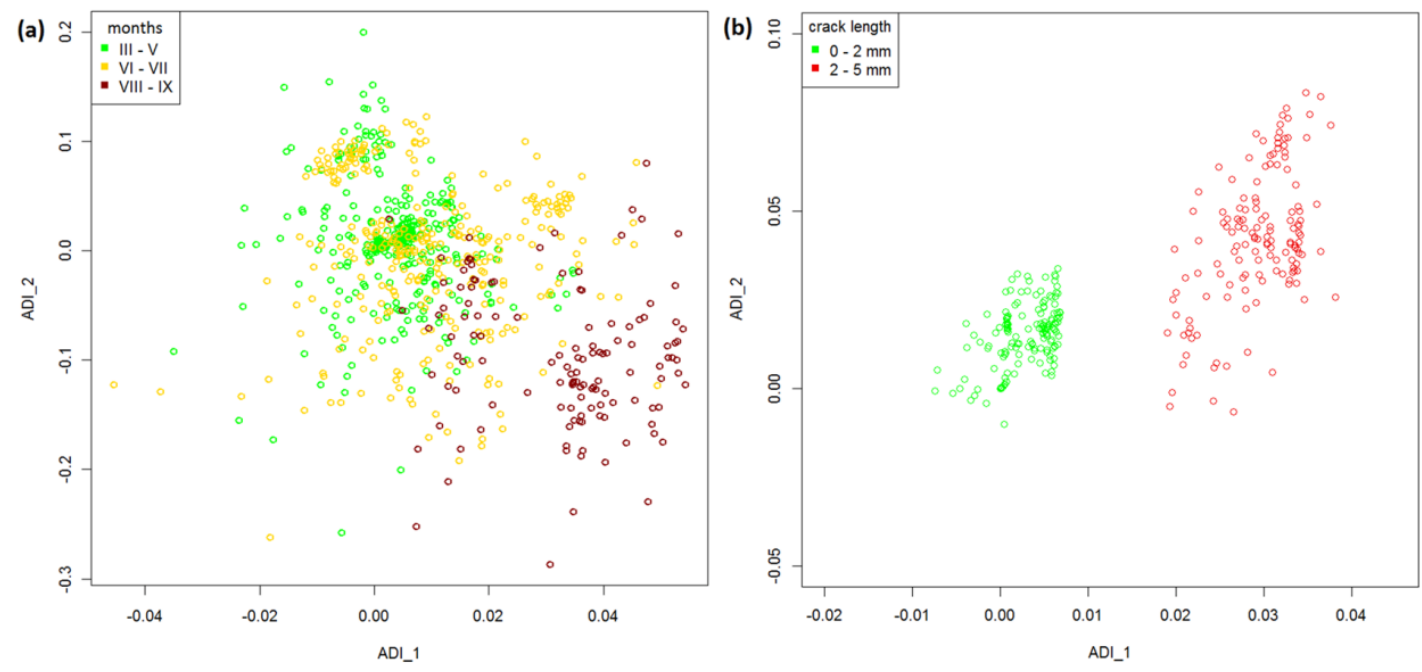

Fig. 8. Averaged Damage Indices for the monitored hot spots

\section{SUMMARY}

This work presents an approach to the development of a SHM system intended for use in a real aircraft structure, including creating the concept of the system, deployment of the sensor network in the aircraft, and remote monitoring of the aircraft structure as well as of structural elements. The system involves the use of the proper mathematical methods for damage detection andthe classification of damage presence and size. The methods proposed in the article were verified during the tests and will be employed to build the system software. Some tests are still in progress and further validation of the elaborated methods will be required. The results of the tests offer great promise for further development of the system concept especially from the point of view of the integration of the sensor technology with the structure, and the methods for data classification and the system's self diagnostics.

\section{ACKNOWLEDGMENTS}

This work was partly supported with the European Union funds by the European Social Fund through the Project "Rozwój nauki - rozwojem regionu - stypendia i wsparcie towarzyszące dla mazowieckich doktorantów".

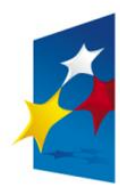

KAPITAK LUDZKI

NARODOWA STRATEGIA SPÓJNOŚCI
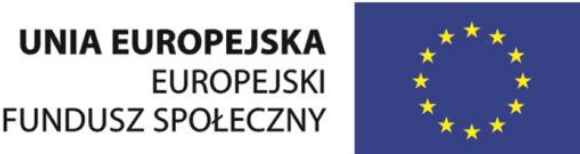

\section{REFERENCES}

1. Z. Su and L. Ye, Identification of Damage Using Lamb Waves, Springer-Verlag, Germany, 2009.

2. D.N. Alleyne and P. Cawley, The excitation of Lamb waves in pipes using dry-coupled piezoelectric transducers, J. Nondestruct. Eval. Vol. 15(1) (1996), p. 11. 
3. P.S. Tua, S.T. Quek and Q. Wang, Detection of cracks in cylindrical pipes and plates using piezo-actuated Lamb waves, Smart Mater. Struct. Vol. 14 (2005), p. 1325.

4. Y. H. Kim, D.H. Kim, J.H. Han and C.G. Kim, Damage assessment in layered composites using spectral analysis and Lamb wave, Compos. Part B-Eng. Vol. 38 (2007), p. 800.

5. Z. Su, L. Ye and Y. Lu, Guided Lamb waves for identification of damage in composite structures: a review, J. Sound Vib. Vol. 295 (2006), p. 753.

6. Q.W. Wang and B.X. Sun, Structural damage localization and quantification using static test data, Struct. Health Monit. Vol. 10(4) (2010), p. 381.

7. T.R. Hay, R.L. Royer, H. Gao, X. Zhao and J.L. Rose, A comparison of embedded sensor Lamb wave ultrasonic tomography approaches for material loss detection, Smart Mater. Struct. Vol. 15(4) (2006), p. 946.

8. D. Wang, L. Ye, Y. Lu and Z. Su, Probability of the presence of damage estimated from an active sensor network in a composite panel of multiple stiffeners, Compos. Sci. Technol. Vol. 69(13) (2009), p. 2054. 